\title{
Efficient delivery of miR-122 to regulate cholesterol metabolism using a non-covalent peptide-based strategy
}

\author{
LILIN WANG ${ }^{1,2^{*}}$, WEI TANG ${ }^{3 *}$, SHIRONG YAN ${ }^{3}$, LONG ZHOU $^{4}$, TAO SHEN $^{2}$, \\ XIUQING HUANG ${ }^{2}$, LIN DOU ${ }^{2}$, MO WANG $^{2}$, SONGLIN YU $^{2}$ and JIAN LI ${ }^{1,2}$ \\ ${ }^{1}$ Center for Gene Diagnosis, Zhongnan Hospital, Wuhan University, Wuhan, Hubei 430071; \\ ${ }^{2}$ The Key Laboratory of Geriatrics, Beijing Institute of Geriatrics and Beijing Hospital, Ministry of Health, Dongdan, \\ Beijing 100730; ${ }^{3}$ Life Science Research Institute of Taihe Hospital, Hubei University of Medicine, Shiyan, Hubei 442000; \\ ${ }^{4}$ Department of Medicine, Shenzhen Family Planning Service Center, Shenzhen, Guangdong 518028, P.R. China
}

Received March 10, 2013; Accepted September 6, 2013

DOI: $10.3892 / \mathrm{mmr} .2013 .1691$

\begin{abstract}
MicroRNAs (miRNAs) are small non-coding RNAs that are important in the pathogenesis of multiple diseases and, therefore, may represent a novel class of targets for therapeutic intervention. However, like the majority of oligonucleotide-based strategies, there are obstacles to their clinical application, including poor cellular uptake due to the low permeability of the cell membrane to negatively charged molecules. MPG is a 27-residue peptide vector which contains a hydrophobic domain derived from the fusion sequence of HIV-1 gp41 and a hydrophilic domain derived from the nuclear localization sequence of SV40 T-antigen. MPG is one of the most promising tools for the non-invasive cellular import of oligonucleotides and analogs. In the present study, a non-covalent peptide-based strategy was used for the efficient delivery of the miRNA-122 (miR-122) mimic and inhibitor into mouse liver cell lines, mouse primary hepatocytes and C. elegans, without any associated cytotoxicity. Moreover, high-performance liquid chromatography analysis determined that MPG and MPG $\Delta^{\mathrm{NLS}}$ delivered the miR-122 mimic and inhibitor into mouse liver cells and effectively regulated cholesterol levels. The results demonstrated that MPG family members may be used for the efficient delivery of miR-122 to regulate cholesterol metabolism, and that this cell-penetrating peptide-based technology may be beneficial for further biological applications of RNA therapeutics in vivo.
\end{abstract}

Correspondence to: Professor Jian Li, The Key Laboratory of Geriatrics, Beijing Institute of Geriatrics and Beijing Hospital, Ministry of Health, 1 Dahua Road, Dongdan, Beijing 100730, P.R. China

E-mail: 1ijian@bjhmoh.cn

${ }^{*}$ Contributed equally

Key words: non-covalent peptide-based strategy, MPG, MPG- $\Delta^{\mathrm{NLS}}$, miRNA-122, cholesterol metabolism

\section{Introduction}

MicroRNAs (miRNAs) are small non-coding RNAs that are essential for normal cellular processes and are commonly dysregulated in cell proliferation, differentiation, survival and motility (1). miRNAs, initially transcribed as long primary transcripts (pri-miRNAs), are processed in the nucleus by the RNase III enzyme Drosha to generate 60- to 120-nt-long precursors containing a stem-loop structure, termed pre-miRNAs (2). These precursors, which are exported into the cytoplasm by the nuclear export factor Exportin-5 and the Ran-GTP cofactor, are cleaved by the RNase enzyme Dicer to release the mature miRNAs (3). miRNAs predominantly bind to the 3'UTRs of their target mRNAs (1). This process, requiring only partial matching, leads to translational repression; while target mRNAs with more stringent pairing requirements may be cleaved $(4,5)$. Numerous seminal studies have demonstrated that disease-associated miRNAs may therefore represent a novel class of therapeutic targets. It has been shown that inhibition of miRNA-122 (miR-122) reduced viral load in non-human primates $(6,7)$ and hepatitis $C$ patients, and thus, miRNA modulators may be candidates for therapeutics. However, the predominant limitations of miRNA applications, similar to the majority of antisense- or nucleic-acid-based strategies, are the charge density, molecular weight and instability in the presence of nucleases. Furthermore, intracellular accumulation and endosomal escape remain to be significant barriers in the delivery of these macromolecules.

Numerous delivery vectors, viral and non-viral, have been developed to facilitate cellular uptake. One group of non-viral vectors that is increasingly utilized for the delivery of various cargoes is the cell-penetrating peptides (CPPs). Over the past 20 years, CPPs have been successfully applied in vitro and in vivo to trigger the movement of a large panel of cargoes, including plasmid DNA, oligonucleotides, siRNA, RNA, proteins, peptides, liposomes and nanoparticles, across the cellular membrane into the cytoplasm of cells (8). CPPs are subdivided into two predominant classes, the first requiring chemical linkage with the cargo (9-12) and the second involving the formation of stable, non-covalent complexes (13-16). MPG is a promising non-covalent strategy that appears to be 
more appropriate for siRNA delivery and yields a significant biological response (17-20).

MPG is a 27-residue peptide vector which contains a hydrophobic domain derived from the fusion sequence of HIV-1 gp41 and a hydrophilic domain derived from the nuclear localization sequence of SV40 T-antigen. MPG is a bipartite amphipathic peptide derived from the fusion peptide domain of HIV-1 glycoprotein 41 (gp41) protein and the nuclear localization signal (NLS) of SV40 large T antigen, which forms stable non-covalent complexes with siRNAs, increases the stability, promotes the cellular uptake without the requirement for prior chemical covalent coupling and enables robust downregulation of target mRNAs $(17,21)$. The hydrophobic moiety of MPG (GALFLGFLGAAGSTMGA) derived from the HIV-1 gp41, is required for efficient targeting to the cell membrane and cellular uptake. The hydrophilic lysine-rich domain (PKKKRKV) derived from the NLS of the SV40 large $\mathrm{T}$ antigen is involved in the predominant interactions with nucleic acids and is required to improve intracellular trafficking of the cargo. The spacer domain (WSQ), improves the flexibility and the integrity of the hydrophobic and hydrophilic domains (22). A variant of MPG with a single mutation in the NLS (MPG $\Delta^{\mathrm{NLS}}$ ) was designed to favor the rapid release of the siRNA into the cytoplasm, thereby allowing for a greater biological response (17). MPG carriers have been used for the delivery of siRNAs into a large range of cell lines, including adherent cell lines, cells in suspension, cancer cell lines and primary cells, which are not transfected using other non-viral approaches $(21,23,24)$. In addition, the final subcellular localization of the siRNA is dependent on the MPG carrier used.

As the miRNA mimic and inhibitors are chemically similar to antisense oligonucleotides and therapeutic siRNAs, positively charged MPG strategies developed for siRNA delivery may also be applicable to the delivery of miRNAs. The development of clinically relevant miRNA formulations frequently involves a thorough evaluation of existing technologies to identify those that may be utilized for miRNA and its chemistry. It was observed that miR-122, a liver-specific miRNA, is involved in diverse aspects of hepatic function and in the progression of liver diseases. miR-122 was also the first miRNA identified to fine-tune lipid metabolism (25). Notably, inhibition of miR-122 in vivo has pronounced effects on cholesterol and fatty acid metabolism. Using antisense strategies, several studies demonstrated that the antagonism of miR-122 in the liver resulted in sustained decreases in plasma cholesterol levels in mice and non-human primates (25-28). Mice treated with antisense oligonucleotides (ASO) to miR-122 showed $25-35 \%$ reductions in total cholesterol, which reflected decreases in the low-density lipoprotein and high-density lipoprotein fractions (25). In African green monkeys, efficient silencing of miR-122 in the liver was achieved with only three doses of the miR-122 inhibitor, and led to sustained decreases in the total plasma cholesterol without any apparent liver toxicity or histopathological change (7). Furthermore, the prolonged antagonism of miR-122 in chimpanzees, achieved by weekly injections of the miR-122 inhibitor for 12 weeks, also reduced plasma cholesterol by $30 \%$. Notably, the reduction in cholesterol levels persisted for several weeks following treatment, suggesting that miR-122 ASO treatment prolonged the effects on hepatic gene expression and cholesterol metabo- lism. There are respective advantages for the aforementioned approaches; however, all of these modifications have impeded the large-scale production due to the low encapsulation efficiency and low endosomal escape. In the present study, a rapid and inexpensive non-covalent strategy using MPG family members was used to deliver an miR-122 mimic and inhibitor into the NCTC 1469 mouse liver cell line, mouse primary hepatocytes and $C$. elegans. High-performance liquid chromatography (HPLC) analysis demonstrated that MPG $\Delta^{\mathrm{NLS}}$ mediated the delivery of the miR-122 mimic and inhibitor and was able to fine-tune cholesterol levels in NCTC 1469 cells.

\section{Materials and methods}

Peptides and the miR-122 mimic and inhibitor. Peptides were synthesized by solid-phase peptide synthesis using standard 9-fluorenylmethyl oxycarbonyl (Fmoc) chemistry (29). HPLC analysis indicated that the synthetic peptide had a purity of $\geq 95 \%$. The mouse miR-122 sequence was obtained from the miRBase Sequence Database (http://mi-crorna.sanger.ac.uk, Release 18.0). Synthetic miR-122 duplexes were chemically synthesized and supplied by RiboBio Biotechnology (Guangzhou, China). The sequences used were as follows: Sense: 5'-UGGAGUGUGACAAUGGUGUUUG-3' and antisense: 5'-AACACCAUUGUCACACUCCAUU-3' for Mmu-miR-122-5p; sense: 5'-UUCUCCGAACGUGUCACG UTT-3' and antisense: 5'-ACGUGACACGUUCGGAGAATT-3' for the negative control; 5'-CAAACACCAUUGUCA CACUCCA-3' for the miR-122 inhibitor and 5'-CAGUACUUU UGUGUAGUACAA-3' for the 2'Ome, miR-122 inhibitor negative control. The 5'-end of the mmu-miR-122-5p sense strand was modified with Fam and Cy3 dye. Synthetic miR-122 duplexes and fluorescently labeled mmu-miR-122-5p sense strand (5'-Fam and 5'-Cy3) were chemically synthesized and supplied by RiboBio Biotechnology (Guangzhou, China).

Cell culture and MPG-mediated transfection. NCTC 1469 and A549 cell lines were derived from mouse liver cells (American Type culture Collection, Manassas, VA, USA). A549 cells were maintained in Dulbecco's modified Eagle's medium (DMEM) supplemented with $2 \mathrm{mM}$ glutamine, $1 \%$ antibiotics $(10,000 \mathrm{mg} / \mathrm{ml}$ streptomycin and 10,000 IU/ml penicillin) and $10 \%(\mathrm{w} / \mathrm{v})$ fetal bovine serum (FBS, Invitrogen Life Technologies, Carlsbad, CA, USA). NCTC 1469 cells were grown in low glucose DMEM (5 mmol/l glucose; Gibco-BRL, Carlsbad, CA, USA) supplemented with $10 \%$ (v/v) horse serum (Hyclone, Rockford, IL, USA), $100 \mathrm{U} / \mathrm{ml}$ penicillin (Gibco-BRL) and $0.1 \mathrm{mg} / \mathrm{ml}$ streptomycin (Gibco-BRL). Cell lines were cultured at $37^{\circ} \mathrm{C}$ in a humidified atmosphere of $95 \%$ $\mathrm{O}_{2}$ and $5 \% \mathrm{CO}_{2}$. Fluorescent labeling of the miR-122 mimic was performed using Fam or Cy3. For peptide (MPG and MPG $\Delta^{\mathrm{NLS}}$ )-mediated delivery, the miR-122 mimic and inhibitor were incubated with carrier peptide at a molecular ratio of 1:20 in phosphate-buffered saline for $30 \mathrm{~min}$ at $37^{\circ} \mathrm{C}$, and then diluted to the required concentration in $500 \mu \mathrm{l}$ DMEM. Cells, grown to $60 \%$ confluence, were then rinsed twice and overlaid with preformed complexes. Following incubation for $30 \mathrm{~min}$ at $37^{\circ} \mathrm{C}, 1 \mathrm{ml}$ fresh DMEM supplemented with $10 \%$ fetal calf serum was added directly to the cells (without removing the overlay of peptide/miR-122 complexes) and cells were returned 
to the incubator for $48 \mathrm{~h}$. For cellular localization experiments, cells were grown on acid-treated glass coverslips to $60 \%$ confluence and then overlaid with preformed peptide/miR-122 complexes. After $1 \mathrm{~h}$, cells were rinsed twice and the cellular localization of Fam-labeled miR-122 mimic was monitored by fluorescence or confocal microscopy.

MPG-mediated delivery of miR-122 into mouse primary hepatocytes. Hepatocytes were isolated from male C57BL/6J mice (age, 8-12 weeks; Peking University Health Science Center, Haidian, China) with collagenase solution (Worthington Biochemicals, Lakewood Township, NJ, USA), as described previously (30). Following filtration and centrifugation, the isolated hepatocytes were dispersed in pre-warmed William's medium E (Sigma-Aldrich, St. Louis, MO, USA) supplemented with $20 \mathrm{ng} / \mathrm{ml}$ dexamethasone (Sigma-Aldrich), ITS (5 mg/l insulin, $5 \mathrm{mg} / \mathrm{l}$ transferrin, $5 \mu \mathrm{g} / \mathrm{l}$ sodium selenate; Sigma-Aldrich), $10 \mu \mathrm{g} / \mathrm{ml}$ gentamicin (Invitrogen Life Technologies) and 10\% (v/v) FBS, at a density of 300,000 cells/well in collagen-coated 12 -well plates or $5 \times 10^{6}$ cells/90-mm-diameter dish. The cultures were maintained for an additional $24 \mathrm{~h}$ prior to transient transfection or nuclear extract preparation. Subsequent procedures for fluorescence imaging were the same as previously mentioned.

MPG-mediated Cy3-labeled miR-122 delivery into C. elegans. The nematode Caenorhabditis elegans (C.elegans) was provided by the Caenorhabditis Genetics Center (St. Paul, MN, USA). Wild-type worms were incubated in $8 \mu \mathrm{l}$ MPG $\Delta^{\mathrm{NLS}} / \mathrm{miR}-122$ mimic complexes for $10 \mathrm{~min}$ to $24 \mathrm{~h}$ and were washed in $\mathrm{M} 9$ solution (43.6 mM Na $2 \mathrm{HPO}_{4}, 22.0 \mathrm{mM} \mathrm{KH} \mathrm{PO}_{4}, 8.6 \mathrm{mM} \mathrm{NaCl}$ and $18.7 \mathrm{mM} \mathrm{NH}_{4} \mathrm{Cl}$ ). Subsequent to this, the worms were transferred to a fresh nematode growth media plate and were allowed to crawl for several minutes to remove excess fluorescent dye.

RNA extraction and miRNA quantification. Total RNA was extracted from cultured cells using TRIzol ${ }^{\circledR}$ Reagent (Invitrogen Life Technologies) according to the manufacturer's instructions. Stem-loop qPCR was used to quantify mature miRNA. Initially, total RNA $(1 \mu \mathrm{g})$ was reverse-transcribed using specific primers for either miR-122 or U6 in a $10-\mu 1$ reaction volume to synthesize cDNA. The synthesized cDNA was diluted up to $150 \mu \mathrm{l}$, and $6 \mu \mathrm{l} \mathrm{cDNA}$ dilution was added to $10 \mu \mathrm{l}$ of the 2X SYBR-Green PCR master mix (Takara Bio, Inc., Shiga, Japan), with $800 \mathrm{nM}$ of each primer in a total volume of $20 \mu \mathrm{l}$. The reactions were amplified for $15 \mathrm{sec}$ at $95^{\circ} \mathrm{C}$ and $1 \mathrm{~min}$ at $60^{\circ} \mathrm{C}$ for 40 cycles. All reactions were run in triplicate and included no template or reverse transcription controls. The cycle number at which the reaction crossed an arbitrarily placed threshold (CT) was determined, and the relative quantity of miR-122 to U6 RNA was calculated using the $2^{-\Delta \Delta \mathrm{CT}}$ method.

Confocal microscopy. Specimens were observed with a Zeiss confocal microscope (LSM; Carl Zeiss Microimaging, Thornwood, NY, USA) or with a Nikon fluorescent microscope (TE2000U; Nikon, Tokyo, Japan). Image collection from the Nikon microscope was conducted with a Hamamatsu Photonics (Hamamatsu, Japan) C4880 cooled CCD camera and the images were processed with Image Pro-Plus (Media Cybernetics, Bethesda, MD, USA) and Photoshop (Adobe, San Jose, CA, USA).
Cytotoxicity assay. The 3,(4,5-dimethylthiazol-2-yl)-2,5-diphenyltetrazolium bromide tetrazole (MTT) reduction assay was used to asses the cell viability. A549 and NCTC 1469 cells were plated in 24 -well plates ( $3 \times 10^{4} /$ well). Following incubation for $24 \mathrm{~h}$, the cells were treated with increasing concentrations of miR-122 mixed with carrier (MPG or MPG $\Delta^{\mathrm{NLS}}$ ) at a 1:20 molar ratio range of 5-100 $\mathrm{nM}$, for $48 \mathrm{~h}$. MTT $(0.5 \mathrm{mg} / \mathrm{ml}$; Sigma-Aldrich) was added to each well $(200 \mu \mathrm{l} /$ well). Following additional incubation for $4 \mathrm{~h}$, the MTT solution was discarded and $200 \mu \mathrm{l}$ dimethylsulfoxide (Amresco, Solon, OH, USA) was added and the plates were shaken gently. The absorbance was measured on an enzyme-linked immunosorbent assay (ELISA) reader at a wavelength of $490 \mathrm{~nm}$.

Measurement of total cholesterol levels in the cultured cells. Cholesterol concentrations of the cultured cells were analyzed by HPLC according to the method by Dong et al (31). Briefly, cholesteryl esters were hydrolyzed with alcoholic potassium hydroxide and, in the presence of an internal standard (stigmasterol), extracted with hexane. The sterols were oxidized to 4-en-3,6-diones with chromic acid and analyzed by HPLC. The low detection limit of the method allowed the measurement of cholesterol in dilute samples and the internal standard calibration eliminated the requirement for volumetric reconstitution of the ultracentrifugation (UC) bottom fractions. Peak area ratios of cholesterol to stigmasterol for the calibrators were linearly regressed on the corresponding cholesterol concentrations and the resulting equation was used to calculate the cholesterol concentrations in the cultured cells.

Statistical analysis. The experiments were repeated at least three times with a minimum sample size of three. Student's t-test was conducted using STATA statistical software (StataCorp LP, College Station, TX, USA), to test for statistical differences between samples. $\mathrm{P}<0.05$ was considered to indicate a statistically significant difference.

\section{Results}

Transfection efficiency and impact on cellular viability of $M P G$ in comparison with MPG $\Delta^{N L S}$. The transfection efficiency and impact on cellular viability of MPG compared with that of MPG $\Delta^{\mathrm{NLS}}$ was determined. Peptide-based mimic and inhibitor delivery was conducted by incubating the NCTC 1469 cells with a constant quantity of miR-122 (50 nM) and increasing volumes of peptides. qPCR analysis demonstrated that the expression of cellular miR-122 gradually and markedly increased in the NCTC 1469 cells transfected with $50 \mathrm{nM}$ of peptide-based miR-122 mimic (Fig. 1A). By contrast, a significant reduction of miR-122 levels was observed in the NCTC 1469 cells treated with peptide-based miR-122 inhibitor (Fig. 1B), indicating that silencing was specific to miR-122. Moreover, it was demonstrated that the expression of miR-122 varied significantly when cells were treated with MPG $\Delta^{\mathrm{NLS}} / \mathrm{mimic}$ or inhibitor, particularly when the molar ratio was higher than 10:1. However, the effective concentration of miR-122 mimic and inhibitor used in biological studies varies, and the concentration commonly used is $<100 \mathrm{nM}$. Therefore, the cytotoxicity of the concentrations of peptide/miRNA complexes were determined by the MTT assay. As shown 
A

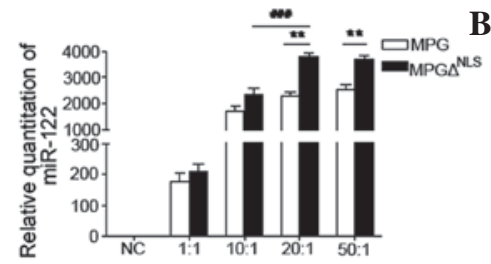

C

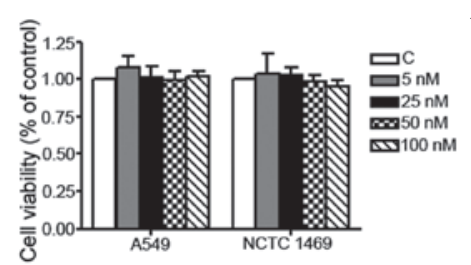

B
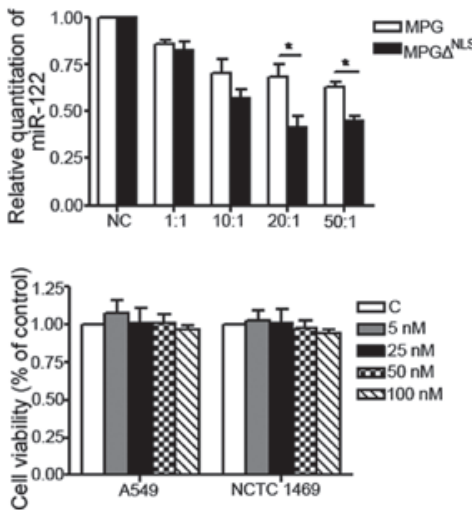

Figure 1. Transfection efficiency and cytotoxicity of peptide-based miR-122 mimic and inhibitor delivery in NCTC 1469 cells. The miR-122 levels were (A) strongly increased in NCTC 1469 cells treated with $50 \mathrm{nM}$ peptide/miR-122 mimic complexes and (B) decreased in NCTC 1469 cells treated with $50 \mathrm{nM}$ peptide/miR-122 inhibitor complexes (molar ratio 1:1-50:1) for $48 \mathrm{~h}$. Relative levels of miR-122 were assessed by miRNA-specific qPCR. (C) Cytotoxicity of MPG/miR-122 mimic complexes and (D) MPG $\Delta^{\mathrm{NLS}} / \mathrm{miR}-122$ mimic complexes at molar ratio 20:1 in NCTC 1469 and A549 cells was evaluated by an MTT assay. Significant differences were determined at ${ }^{*} \mathrm{P}<0.05$ and ${ }^{* *} \mathrm{P}<0.01$ between MPG and MPG $\Delta^{\mathrm{NLS}}$, and ${ }^{\# \# \#} \mathrm{P}<0.001$ between molar ratio 10:1 to $20: 1$ of $\mathrm{MPG} \Delta^{\mathrm{NLS}} / \mathrm{miR}-122$ mimic complexes. Data are presented as the mean $\pm \mathrm{SEM}, \mathrm{n}=3$ independent experiments. miR-122, miRNA-122.

in Fig. 1C and D, the viability of NCTC 1469 and A549 cells remained unaffected when the cells were exposed to peptide/miRNA complexes at a concentration of $100 \mathrm{nM}$. These results demonstrate that $>100 \mathrm{nM}$ of peptide/miRNA complexes had no significant cytotoxic effect on mammalian cells. Thus, neither of the complexes were toxic at any of the concentrations tested, as indicated by the cell viability assays.

Cellular localization of peptidelmiR-122 mimic complexes. A recommended dosage for a silencing response is $50 \mathrm{nM}$ miRNA. The optimal incubation time of Fam-labeled miR-122 mimic required for detection using confocal microscopy was determined. The confocal microscopy characterization demonstrated that, following incubation for $10 \mathrm{~min}$ with MPG/miR-122 mimic and MPG $\Delta^{\mathrm{NLS}} / \mathrm{miR}-122$ mimic, punctuated fluorescence was observed in the cytoplasm of A549 cells, indicating the entry of the complexes into the cells. Additionally, with increasing incubation time, the fluorescence appeared to aggregate to more discrete areas in the cytoplasm (data not shown). MPG $\Delta^{\mathrm{NLS}}$, a sequence variant of MPG, contains a single mutation of the second lysine residue to serine (KSKRKV) in the NLS motif. This mutation has previously been observed to markedly increase the import of NLS-containing siRNA into the cytoplasm (17). As shown in Fig. 2A, green fluorescence was observed in the cytoplasm and nucleus, while in Fig. 2B, the complexes appeared to be heterogeneously distributed in the cytoplasm of A549 cells.

$M P G \Delta^{N L S}$-mediated delivery of Cy3-labeled miR-122 mimic in mouse primary hepatocytes. To identify the cellular location of the miRNA, the intracellular delivery of MPG $\Delta^{\mathrm{NLS}}$ in mouse primary hepatocytes and NCTC 1469 cells was investigated. An alternate fluorescent probe (Cy3) was used in order to avoid any artifacts which may have been associated with the nature of the probe. The majority of the Cy3-labeled miR-122 mimic (red dots) was localized in the cytoplasm, demonstrating that MPG $\Delta^{\mathrm{NLS}}$ may also be used for the efficient delivery of the miR-122 mimic into the NCTC 1469 cells (Fig. 3A) and mouse primary hepatocytes (Fig. 3B).
$M P G \Delta^{N L S}$-mediated miR-122 mimic delivery in model organisms. To assess the MPG $\Delta^{\mathrm{NLS}}$-mediated miR-122 mimic delivery in model organisms, the fluorescence concentration in model organisms soaked with Cy3 labeled MPG $\Delta^{\mathrm{NLS}}$-mediated miR 122 mimic was determined using a fluorescence microscope. As shown in Fig. 4, a markedly higher concentration of fluorescence was detected following treatment with MPG $\Delta^{\mathrm{NLS}}$ (Fig. 4B), while fluorescence in the naked Cy3-labeled miR-122 mimic was faint (Fig. 4A).

Effect of MPG $\Delta^{N L S} / \mathrm{miR}-122$ mimic and inhibitor on cholesterol levels. As miR-122 regulates cholesterol levels (31), the levels of cholesterol were determined following transfection with MPG $\Delta^{\mathrm{NLS}} / \mathrm{miR}-122 \mathrm{mimic}$ and MPG $\Delta^{\mathrm{NLS}} / \mathrm{miR}-122$ inhibitor in NCTC 1469 cells. It was demonstrated that cholesterol levels were increased following transfection with the MPG $\Delta^{\mathrm{NLS}} / \mathrm{miR}-122$ mimic (Fig. 5A). Conversely, transfection with the MPG $\Delta^{\mathrm{NLS}} / \mathrm{miR}-122$ inhibitor decreased cholesterol levels in NCTC 1469 cells (Fig. 5B). This suggested the efficient delivery of miR-122 regulated cholesterol metabolism through the non-covalent peptide-based strategy.

\section{Discussion}

For the past two decades, studies have investigated the application of oligonucleotides as therapeutic agents. Theoretically, the administration of an miRNA mimic compound would replenish miRNA activity and concomitantly restore negative regulation of multiple target genes. By contrast, an miRNA inhibitor compound would antagonize miRNA activity and thereby relieve the inhibition of target genes, effectively adjusting the function of cells. However, as oligonucleotides are negatively charged hydrophilic macromolecules, their delivery is problematic. MPG is a 27-residue amphipathic peptide, which forms stable particles with siRNA and improves siRNA delivery ex vivo and in vivo without activating the innate immune response $(13,32,33)$. siRNA and miRNA exhibit similar chemical properties and therefore, MPG strategies that are used for siRNA delivery may also be used for miRNA delivery. 


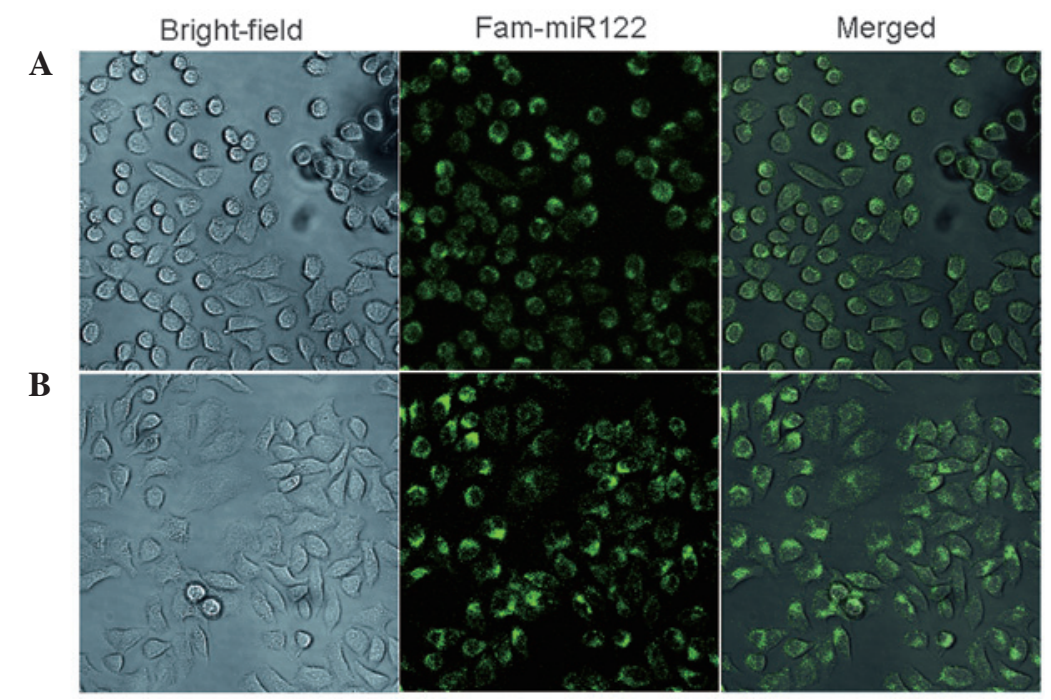

Figure 2. Cellular localization of peptide/miR-122 mimic complexes. A549 cells were transfected with complexes of (A) $1 \mathrm{mM}$ MPG and $50 \mathrm{nM}$ Fam-labeled miR-122 mimic and with complexes of (B) $1 \mathrm{mM} \mathrm{MPG} \Delta^{\mathrm{NLS}}$ and $50 \mathrm{nM}$ Fam-labeled miR-122 mimic, for $10 \mathrm{~min}$. Microscopic analysis was conducted using a confocal laser scanning microscope (LSM 510, Carl Zeiss; magnification, x200). miR-122, miRNA-122.

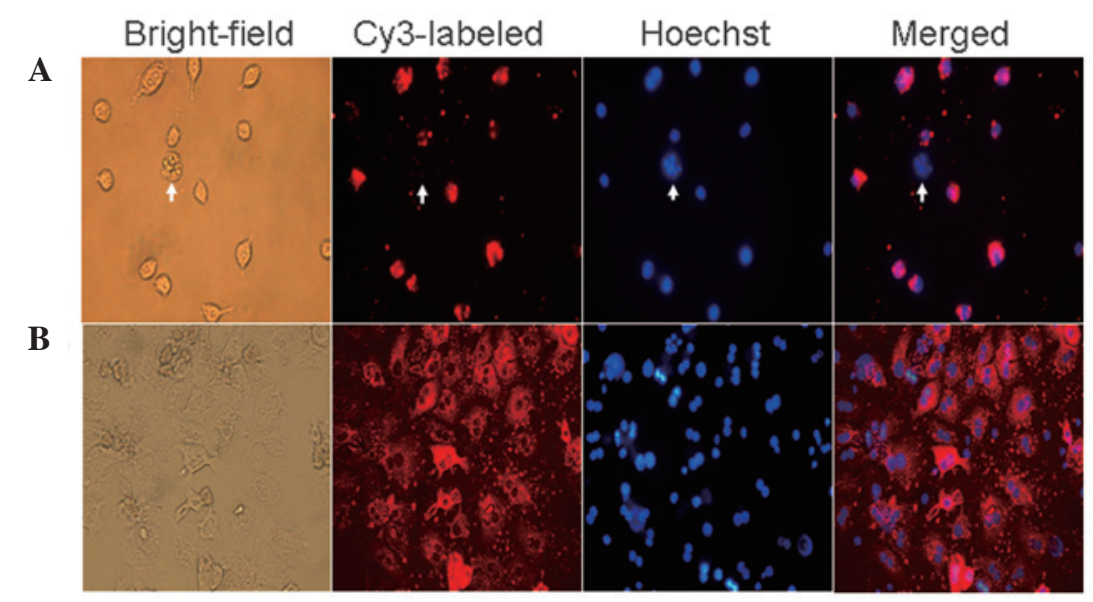

Figure 3. MPG $\Delta^{\mathrm{NLS}}$ mediated delivery of Cy3-labeled miR-122 mimic in mouse primary hepatocytes and NCTC 1469 cells. (A) NCTC 1469 cells and (B) mouse primary hepatocytes were treated with $50 \mathrm{nM}$ Cy3-labeled miR-122 mimic and MPG $\Delta^{\mathrm{NLS}}$ complexes (molar ratio, 20:1) for 10 min. Hoechst 33342 staining revealed the nuclear fragmentation and location of the Cy3-labeled miR-122 mimic mediated by MPG ${ }^{\mathrm{NLS}}$. Arrows indicates apoptotic cell. Microscopic analysis was conducted using a Nikon fluorescent microscope (magnification, x200). miR-122, miRNA-122.

A

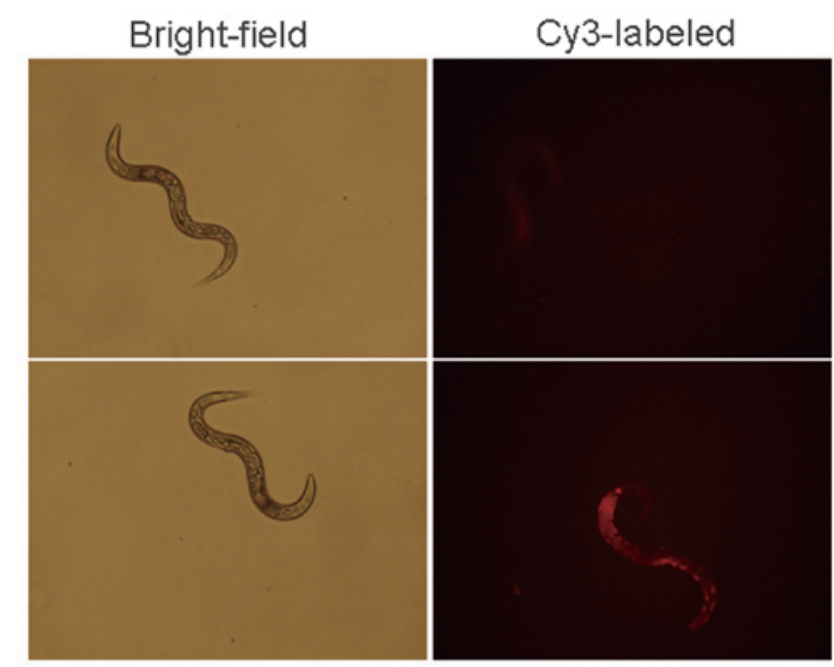

Figure 4. MPG $\Delta^{\mathrm{NLS}}$ mediated Cy3-labeled miR-122 mimic delivery in a model organism. C. elegans were soaked in (A) $50 \mathrm{nM}$ naked Cy3-labeled miR-122 mimic and (B) treated with Cy3-labeled $50 \mathrm{nM} \mathrm{MPG} \Delta^{\mathrm{NLS}} / \mathrm{miR}-122$ mimic complexes (molar ratio, 20:1) for 10 min (magnification, x200). miR-122, miRNA-122. 
A

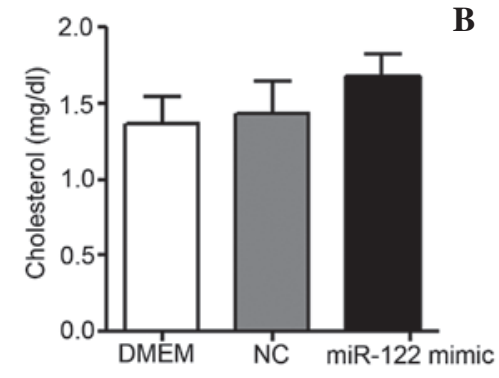

B

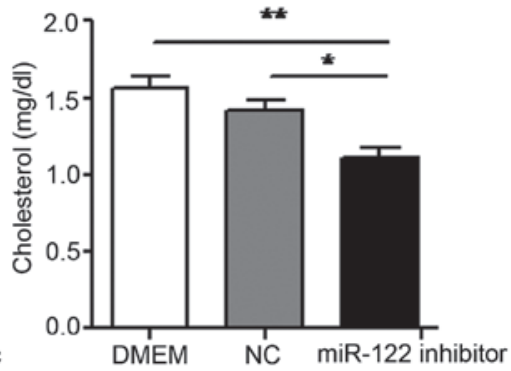

Figure 5. Effect of MPG $\Delta^{\text {NLS}} / \mathrm{miR}-122$ mimic and inhibitor on cholesterol levels in NCTC 1469 cells. The levels of cholesterol in (A) NCTC 1469 cells transfected with $50 \mathrm{nM}$ MPG $\Delta^{\mathrm{NLS}} / \mathrm{miR}-122$ mimic complexes and (B) $50 \mathrm{nM}$ MPG $\Delta^{\mathrm{NLS}} / \mathrm{miR}-122$ inhibitor complexes at molar ratio $20: 1$. Data are presented as the mean \pm SEM, $n=3$ independent experiments. ${ }^{*} \mathrm{P}<0.05$ and ${ }^{* *} \mathrm{P}<0.01$ vs. the control. miR-122, miRNA-122; DMEM, Dulbecco's modified Eagle's medium.

In the present study, MPG family-mediated miR-122 mimic and inhibitor delivery was developed in vitro and in C. elegans. Various technical factors were optimized, such as the molar ratio of peptides/oligonucleotide complexes, and the efficiency of MPG in modulating miR-122 compared with that of MPG $\Delta^{\mathrm{NLS}}$ was determined. Using miRNA-specific $\mathrm{qPCR}$ measurements, the levels of miRNA-122 were observed in NCTC 1469 cells. The results demonstrated that the miR-122 levels increased dose-dependently up to 4000 -fold compared with that of the delivery of the negative control when NCTC 1469 cells were treated with $50 \mathrm{nM}$ peptide/miR-122 mimic complexes at a molar ratio of 20:1 (Fig. 1A). A 60\% reduction of miR-122 levels was obtained in the cells transfected with peptide/miR-122 inhibitor complexes at a molar ratio of 20:1 (Fig. 1B). However, further increase of the peptide/oligonucleotide to a molar ratio of 50:1 demonstrated only a marginal change in the miR-122 levels $(\mathrm{P}>0.05)$, which may be explained by a net increase in the size of particles. At a lower ratio (1:1), the efficiency of MPG- and MPG $\Delta^{\mathrm{NLS}}$-based miR-122 mimic delivery was 10-fold lower than that of peptides at a 20:1 ratio and similar results were observed in the delivery of miR-122 inhibitor at 1:1 ratio. These results are in agreement with those of Crombez et al (34), which may indicate that the complexes were unstable and poorly taken up. By contrast, there was a limited adjustment of the miR-122 levels when the cells were treated with $\mathrm{MPG} / \mathrm{miR}-122$ complexes compared with that of MPG $\Delta^{\mathrm{NLS}}$ at the same peptide/miR-122 ratio. A higher efficiency of MPG $\Delta^{\mathrm{NLS}} / \mathrm{miR}-122$ is associated with a single mutation in the NLS sequence that induces rapid release of the miR-122 into the cytoplasm, and this correlates with an increased biological response. Therefore, to ensure optimal biological conditions for miR-122 delivery, MPG $\Delta^{\mathrm{NLS}} / \mathrm{miR}-122$ particles were used and systematically prepared at a 20:1 ratio.

In order to use MPG derivatives as delivery vehicles for miRNA therapeutics, the degree of cytotoxicity of peptides/miRNA complexes is critical. Thus, the cytotoxicity of MPG and MPG $\Delta^{\mathrm{NLS}}$ with miR-122 mimic complexes was determined by an MTT assay in NCTC 1469 and A549 cells (Fig. 1C and D). Following transfection for $48 \mathrm{~h}$, no toxicity was detected up to a concentration of $100 \mathrm{nM}$ in the cell lines and only $4 \%$ of cell death was observed with $100 \mathrm{nM}$ of MPG/miR-122 particles in NCTC 1469 cells. Therefore, peptide/miRNA was considered to be relatively non-toxic for cells.

Fam-labeled miR-122 mimic was distributed in the cytoplasm and nucleus within $10 \mathrm{~min}$ when the cells were transfected with MPG, but remained predominantly in the cytoplasm of the cells transfected with MPG $\Delta^{\mathrm{NLS}}$, which is consistent with the results of a previous study (17) and contributed to the higher efficiency of downregulation. Moreover, further studies demonstrated that MPG- and MPG $\Delta^{\mathrm{NLS}}$-mediated miR-122 mimic and inhibitor delivery was independent of the presence of serum (data not shown). In order to avoid any artifacts that may have been associated with the nature of the probe, an alternate fluorescent probe (Cy3) was used to label miR-122 mimic in NCTC 1469 cells. Furthermore, while primary cells remained relatively refractory to transfection, MPG $\Delta^{\mathrm{NLS}}$ mediated Cy3-labeled miR-122 mimic transfection within $10 \mathrm{~min}$ at a $95 \%$ efficiency. These results collectively demonstrated that MPG $\Delta^{\mathrm{NLS}}$ may be able to transfect different cell types without affecting the cell phenotype, which opens novel possibilities for large-scale miRNA therapy in disease-relevant primary cells. Meanwhile, it was demonstrated that the fluorescence was markedly low in certain cells (Fig. 3, indicated by an arrow), which may be due to the variation in the membrane components. However, Jones and Howl (35) suggested that CPP induced non-specific membrane perturbations, thus leading to cell death by necrotic mechanisms, particularly at higher concentrations.

In addition to microinjection, soaking $C$. elegans in dsRNA is also an effective method of delivery (36). To investigate the delivery efficiency of MPG $\Delta^{\mathrm{NLS}}$ in model organisms, C. elegans were soaked in the MPG $\Delta^{\mathrm{NLS}}$ and Cy3-labeled miR-122 mimic complexes for varying time periods (10 min-1 day). To avoid artifacts from the ingestion of the fluorescent miR-122, the fluorescence was observed within $10 \mathrm{~min}$ after soaking. Greater fluorescence was detected in the $C$. elegans treated with MPG $\Delta^{\mathrm{NLS}}$ compared with those without peptide treatment. Moreover, fluorescence localization in the C.elegans was monitored after $24 \mathrm{~h}$, which accumulated mainly inside the intestines (data not shown).

To investigate the physiological effects of miR-122 silencing on cholesterol metabolism, the total cholesterol levels in NCTC 1469 cells $48 \mathrm{~h}$ following coculture with MPG $\Delta^{\mathrm{NLS}} / \mathrm{miR}-122$ mimic and inhibitor were determined. To the best of our knowledge, this is the first time that cholesterol levels have been regulated by MPG-mediated miRNA delivery. Using the novel delivery method, the levels of miR-122 in the NCTC 1469 cells were regulated, accompanied by $15-20 \%$ cholesterol changes (Fig. 5). In a previous study, total cholesterol reduced by a similar extent (26-28\%) in mice treated with miR-122 ASO as compared with saline-treated mice (25).In the present study, 
there were no chemical modifications that were associated with lower stability of the complexes and lower pharmacological efficiency.

However, numerous challenges remain in the development of MPG derivative-based RNA therapeutics. Future studies are required to calculate and analyze $3 \mathrm{D}$ models of the non-covalent MPG/RNA complexes in order to understand their formation and stabilization. Further toxicity studies of the chemically modified miRNA inhibitor in animal models are also required.

In conclusion, the non-covalent peptide-based strategy was used for the efficient delivery of miR-122 mimic and inhibitor into mouse liver cell lines, as well as mouse primary hepatocytes and C.elegans, without any associated cytotoxicity. This method is valuable in the study of the biological functions of individual miRNAs and miRNA-associated gene-regulatory networks, and in evaluating miRNA targets. This method is significantly faster and simpler than comparable genetics-based approaches.

\section{Acknowledgements}

This study was supported in part by the open funding of the National Key Laboratory of Crop Genetic Improvement (grant no. ZK201201) and by funding from the Guangdong Provincial Population and Family Planning Commission of scientific research project (grant no. 20110223). The authors would like to thank Professor Jian-Ping Cai and Dr. Xiaoyang Zhou (The Key Laboratory of Geriatrics, Beijing Hospital and Beijing Institute of Geriatrics) for providing the nematode Caenorhabditis elegans (C. elegans) and Dr. Jun Dong (The Peking Union Medical College and Chinese Academy of Medical Sciences) for assistance with the HPLC analysis.

\section{References}

1. Bartel DP: MicroRNAs: genomics, biogenesis, mechanism, and function. Cell 116: 281-297, 2004.

2. Lee Y, Ahn C, Han J, et al: The nuclear RNase III Drosha initiates microRNA processing. Nature 425: 415-419, 2003

3. Yi R, Qin Y, Macara IG and Cullen BR: Exportin-5 mediates the nuclear export of pre-microRNAs and short hairpin RNAs. Genes Dev 17: 3011-3016, 2003.

4. Ambros V: The functions of animal microRNAs. Nature 431: 350-355, 2004

5. Yekta S, Shih IH and Bartel DP: MicroRNA-directed cleavage of HOXB8 mRNA. Science 304: 594-596, 2004.

6. Hildebrandt-Eriksen ES, Aarup V, Persson R, Hansen HF, Munk ME and Orum H: A locked nucleic acid oligonucleotide targeting microRNA 122 is well-tolerated in cynomolgus monkeys. Nucleic Acid Ther 22: 152-161, 2012.

7. Elmén J, Lindow M, Schutz S, et al: LNA-mediated microRNA silencing in non-human primates. Nature 452: 896-899, 2008.

8. Heitz F, Morris MC and Divita G: Twenty years of cell-penetrating peptides: from molecular mechanisms to therapeutics Br J Pharmacol 157: 195-206, 2009.

9. Zatsepin TS, Turner JJ, Oretskaya TS and Gait MJ: Conjugates of oligonucleotides and analogues with cell penetrating peptides as gene silencing agents. Curr Pharm Des 11: 3639-3654, 2005.

10. El-Andaloussi S, Holm T and Langel U: Cell-penetrating peptides: mechanisms and applications. Curr Pharm Des 11: 3597-3611, 2005.

11. Joliot A and Prochiantz A: Transduction peptides: from technology to physiology. Nat Cell Biol 6: 189-196, 2004.

12. Torchilin VP: Tat peptide-mediated intracellular delivery of pharmaceutical nanocarriers. Adv Drug Deliv Rev 60: 548-558, 2008.
13. Deshayes S, Morris M, Heitz F and Divita G: Delivery of proteins and nucleic acids using a non-covalent peptide-based strategy. Adv Drug Deliv Rev 60: 537-547, 2008

14. Deshayes S, Morris MC, Divita G and Heitz F: Cell-penetrating peptides: tools for intracellular delivery of therapeutics. Cell Mol Life Sci 62: 1839-1849, 2005

15. Snyder EL and Dowdy SF: Recent advances in the use of protein transduction domains for the delivery of peptides, proteins and nucleic acids in vivo. Expert Opin Drug Deliv 2: 43-51, 2005.

16. Eguchi A and Dowdy SF: siRNA delivery using peptide transduction domains. Trends Pharmacol Sci 30: 341-345, 2009.

17. Simeoni F, Morris MC, Heitz F and Divita G: Insight into the mechanism of the peptide-based gene delivery system MPG: implications for delivery of siRNA into mammalian cells. Nucleic Acids Res 31: 2717-2724, 2003.

18. Yoo JW, Hong SW, Kim S and Lee DK: Inflammatory cytokine induction by siRNAs is cell type- and transfection reagent-specific. Biochem Biophys Res Commun 347: 1053-1058, 2006.

19. Crombez L, Charnet A, Morris MC, Aldrian-Herrada G, Heitz F and Divita G: A non-covalent peptide-based strategy for siRNA delivery. Biochem Soc Trans 35: 44-46, 2007.

20. Lundberg P, El-Andaloussi S, Sutlu T, Johansson H and Langel U: Delivery of short interfering RNA using endosomolytic cell-penetrating peptides. FASEB J 21: 2664-2671, 2007.

21. Simeoni F, Morris MC, Heitz F and Divita G: Peptide-based strategy for siRNA delivery into mammalian cells. Methods Mol Biol 309: 251-260, 2005

22. Morris MC, Chaloin L, Méry J, Heitz F and Divita G: A novel potent strategy for gene delivery using a single peptide vector as a carrier. Nucleic Acids Res 27: 3510-3517, 1999.

23. Morris KV, Chan SW, Jacobsen SE and Looney DJ: Small interfering RNA-induced transcriptional gene silencing in human cells. Science 305: 1289-1292, 2004

24. Nguyen QN, Chavli RV, Marques JT, et al: Light controllable siRNAs regulate gene suppression and phenotypes in cells. Biochim Biophys Acta 1758: 394-403, 2006.

25. Esau C, Davis S, Murray SF, et al: miR-122 regulation of lipid metabolism revealed by in vivo antisense targeting. Cell Metab 3: 87-98, 2006.

26. Liu GT, Carrazana EJ, Macklis JD and Mikati MA: Delayed oculogyric crises associated with striatocapsular infarction. J Clin Neuroophthalmol 11: 198-201, 1991.

27. Esau CC: Inhibition of microRNA with antisense oligonucleotides. Methods 44: 55-60, 2008.

28. Lanford RE, Hildebrandt-Eriksen ES, Petri A, et al: Therapeutic silencing of microRNA-122 in primates with chronic hepatitis C virus infection. Science 327: 198-201, 2010.

29. Méry J, Granier C, Juin M, and Brugidou J: Disulfide linkage to polyacrylic resin for automated Fmoc peptide synthesis. Immunochemical applications of peptide resins and mercaptoamide peptides. Int J Pept Protein Res 42: 44-52, 1993.

30. Salonpää P, Pelkonen O, Kojo A, Pasanen M, Negishi M and Raunio H: Cytochrome P4502A5 expression and inducibility by phenobarbital is modulated by cAMP in mouse primary hepatocytes. Biochem Biophys Res Commun 205: 631-637, 1994.

31. Dong J, Guo H, Yang R, et al: Serum LDL- and HDL-cholesterol determined by ultracentrifugation and HPLC. J Lipid Res 52: 383-388, 2011.

32. Krützfeldt J, Rajewsky N,Braich R, et al: Silencing of microRNAs in vivo with 'antagomirs'. Nature 438: 685-689, 2005.

33. Crombez L, Morris MC, Heitz F and Divita G: A non-covalent peptide-based strategy for ex vivo and in vivo oligonucleotide delivery. Methods Mol Biol 764: 59-73, 2011.

34. Crombez L, Morris MC, Dufort S, et al: Targeting cyclin B1 through peptide-based delivery of siRNA prevents tumour growth. Nucleic Acids Res 37: 4559-4569, 2009.

35. Jones S and Howl J: Applications of cell-penetrating peptides as signal transduction modulators for the selective induction of apoptosis. Methods Mol Biol 683: 291-303, 2011.

36. Kimber MJ, McKinney S, McMaster S, Day TA, Fleming CC and Maule AG: flp gene disruption in a parasitic nematode reveals motor dysfunction and unusual neuronal sensitivity to RNA interference. FASEB J 21: 1233-1243, 2007. 